https://helda.helsinki.fi

\title{
Enzymatic assay for urine lactose in the assessment of recent intravenous abuse of buprenorphine
}

Keltanen, Terhi N.

2019-09

Keltanen , T N , Heikman , P K, Muhonen , L H , Gunnar , T O \& Ojanperä , I A 2019 , '

Enzymatic assay for urine lactose in the assessment of recent intravenous abuse of buprenorphine ' , Drug Testing and Analysis , vol. 11 , no. 9 , pp. 1412-1418 . https://doi.org/10.1002/dta.2654

http://hdl.handle.net/10138/312966

https://doi.org/10.1002/dta.2654

acceptedVersion

Downloaded from Helda, University of Helsinki institutional repository.

This is an electronic reprint of the original article.

This reprint may differ from the original in pagination and typographic detail.

Please cite the original version. 
Keltanen Terhi (Orcid ID: 0000-0002-7668-8329)

Ojanperä Ilkka (Orcid ID: 0000-0001-5790-1473)

\section{Enzymatic Assay for Urine Lactose in Assessment of Recent Intravenous Abuse of}

\section{Buprenorphine}

Terhi N. Keltanen ${ }^{1,2 *}$, Pertti K. Heikman ${ }^{3,4}$, Leea H. Muhonen ${ }^{4}$, Teemu O. Gunnar ${ }^{1}$, Ilkka A. Ojanperä ${ }^{1,2}$

1) Forensic Toxicology Unit, National Institute for Health and Welfare, Helsinki, Finland

2) Department of Forensic Medicine, University of Helsinki, Helsinki, Finland

3) University of Helsinki, Psychiatry, Helsinki, Finland

4) Helsinki University Central Hospital, Psychiatry, Helsinki, Finland

*Corresponding Author:

Terhi Keltanen

Forensic Toxicology Unit, National Institute for Health and Welfare

P.O. Box 30

00271 Helsinki, Finland

Tel: +358405731128

Fax: +358 295248713

E-mail: terhi.keltanen@helsinki.fi

Declarations of competing interest

Terhi Keltanen received a research grant from Yrjö Jahnsson Foundation for this study. None of the authors have any other competing interests to declare.

Keywords: opioid dependence, buprenorphine, intravenous abuse, urine lactose, enzymatic

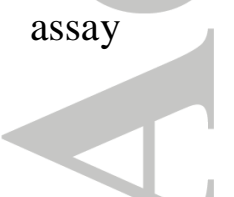

This article has been accepted for publication and undergone full peer review but has not been through the copyediting, typesetting, pagination and proofreading process which may lead to differences between this version and the Version of Record. Please cite this article as doi: 10.1002/dta.2654 


\begin{abstract}
Background

Laboratory methods for differentiating intravenous substance abuse from medical oral or sublingual use are lacking. The disaccharides lactose and sucrose are common excipients in both medical preparations and illicit drugs. If substances are abused intravenously, the disaccharides are excreted unchanged in urine. This study aimed to evaluate whether urine lactose indicates recent intravenous abuse of substances with special reference to buprenorphine.

Methods

Samples were attained from 21 patients in assessment for opioid maintenance treatment (OMT) and from 29 patients already enrolled in OMT. Patients were divided in two groups. Group 1 patients reported intravenous abuse of buprenorphine within 48 hours and Group 2 more than 48 hours since the last injection. The urine samples were assayed for lactose using an enzymatic method. To verify intravenous abuse, examination of needle marks and mass spectrometric analysis of abused substances were performed.

\section{Results}

Urine lactose concentration was significantly higher in Group $1(86 \mathrm{mg} / \mathrm{l})$ than in Group 2 (24 $\mathrm{mg} / \mathrm{l})$. All samples with lactose concentration more than $140 \mathrm{mg} / \mathrm{l}$ were given by the Group 1 patients, who all had recent injection marks. A significant inverse correlation was found between the time from last injection and the urine lactose concentration within the 48- hour post-injection period.

\title{
Conclusions
}

Elevated lactose concentrations in urine could indicate intravenous abuse of substances containing lactose. The co-occurrence of higher than $140 \mathrm{mg} / \mathrm{l}$ of lactose in urine by means of enzymatic assay verified by fresh puncture sites is a potential indicator of recent intravenous abuse of buprenorphine. 


\section{Introduction}

Opioid maintenance treatment (OMT) aims at preventing or significantly reducing illicit opioid abuse, drug-related crimes, intravenous substance abuse, and risks associated with overdose $^{1-4}$. It enables the treatment of opioid dependence and improves patients' psychosocial welfare ${ }^{l}$. The need for efficient OMT has been emphasized lately, especially in the context of the opioid crisis in the United States ${ }^{5-7}$. Yet OMT may be underutilized by physicians due to financial reasons, lack of time for more patients, or lack of belief in the benefits of $\mathrm{OMT}^{7,8}$. The most commonly used OMT medication is either methadone (MET) or buprenorphine, the latter being administered in the form of mono-buprenorphine (BPN) or the combination preparation buprenorphine-naloxone $(\mathrm{BNX})^{3,4}$. The opioid antagonist naloxone in BNX may reduce the risk of diversion and intravenous abuse ${ }^{4}$, but it does not prevent these actions completely ${ }^{9-11}$ among tablet or film users ${ }^{12}$. Injection use of buprenorphine is the most biologically efficient route of administration in terms of bioavailability ${ }^{11}$. The most important reasons for injecting BPN/BNX are to treat addiction and alleviate withdrawal ${ }^{9-11}$. Furthermore, the decision to inject buprenorphine may be influenced by cost, as smaller doses can be used in comparison to sublingual dosing ${ }^{11}$

Thus, despite the individual and societal benefits attained with OMT, abuse and diversion of OMT drugs still occur ${ }^{13}$. In addition, polydrug abuse is a known problem among OMT patients, and it appears to be related to insufficient dosage of OMT drugs ${ }^{14,15}$ Through existing modern laboratory diagnostics, it is possible to reveal the abuse of illicit drugs, allowing appropriate intervention to improve the outcome of OMT and the health of patients $16,17$.

Parenteral administration of substances cannot usually be differentiated by ordinary drug testing procedures from adequate oral or sublingual administration. However, the OMT 
medications, as well as several other medicines and also illicit drugs, often contain disaccharides as excipients ${ }^{18}$. Both the BPN and BNX sublingual tablets contain lactose, and some methadone preparations contain sucrose and lactose. When ingested, lactose is hydrolysed to its components glucose and galactose, in order to be absorbed intestinally and further transferred in to blood circulation ${ }^{19}$. If the hydrolysis is impaired, lactose will be fermented in to gas and short chain fatty acids in the colon. Therefore, in normal circumstances lactose is not excreted in urine as such, but as its metabolite monosaccharides. Lactose may be excreted in urine as such in cases of lactose malabsorption ${ }^{19-21}$, or when prolactin concentrations are elevated, as in pregnancy, breastfeeding or hyperprolactinemia 22. The levels of lactose in these circumstances vary, and depend on the method and units used. Analysis of disaccharides has been utilized to resolve the route of administration of substances with both clinical ${ }^{23}$ and post-mortem (PM) ${ }^{24}$ urine samples.

In the present study we apply the simple enzymatic method in an actual clinical setting to the analysis of urine lactose in opioid-dependent patients prior to and during OMT. The main aim of this study is to assess if the urine lactose might be an indicator of recent intravenous abuse of buprenorphine or other licit or illicit substances among opioid dependent patients. To verify the laboratory findings, examination of the whole body for injection marks were examined concomitantly with the urine sample collection.

\section{Materials and methods}

\section{Patient recruitment}

The opioid-dependent (DSM-IV, Diagnostic and Statistical Manual of Mental Disorders, Fourth Edition) patients were either in assessment of the need for OMT or already enrolled in OMT at the outpatient clinic for opioid-dependent patients of Helsinki University Central 
Hospital (HUCH). Fifty-three of the 112 patients (46.1\%) gave informed consent for the study. One patient later withdrew consent, and in two cases urine samples were not obtained from patients who had given informed consent. Thus, 50 patients participated in the study. Thirty-seven of these patients also gave a second urine sample at another testing time, as the patients are tested in the clinic regularly when in OMT or in evaluation for it. In total, there were 87 samples for laboratory analyses. Forty-one urine samples were collected from 21 patients who were in evaluation for OMT, and 46 samples were collected from 29 patients already in OMT.

The study was approved by the HUCH Ethics Committee for gynecology and obstetrics, pediatrics, and psychiatry (record number HUS/1518/2016). The study was conducted in accordance with the 1964 Declaration of Helsinki. All study participants were able to read and understand the patient information sheet and had signed the informed consent form. The participants were free to discontinue their participation in the study at any point.

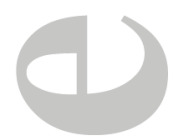

OMT medications of study patients

The OMT patients were medicated either with MET or BNX. The BNX medication was Suboxone sublingual tablet containing buprenorphine and naloxone in a ratio of 4:1 (Reckitt Benckiser Healthcare Ltd., Slough, UK). This sublingual tablet contained also lactose monohydrate, mannitol, corn starch, Povidone K 30, citric acid, anhydrous sodium citrate magnesium stearate, acesulfame potassium, and natural lemon and lime flavours. The MET medication, produced by the HUCH pharmacy, was an oral methadone solution $(5 \mathrm{mg} / \mathrm{mL})$ containing also methylparahydroxybenzoate. The solution did not contain lactose. 
Clinical examination and interview

The patients were examined for both recent and old needle marks, and for this purpose the whole body was undressed except for underwear. The analysis of recent and old injection marks was carried out according to the WHO guidelines ${ }^{l}$, i.e. recent injection marks are small and red, and are sometimes inflamed or surrounded by slight bruising. Old injection sites are generally not inflamed, but sometimes show pigmentation changes (either lighter or darker), and the skin may have atrophied, giving a sunken impression.

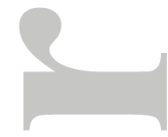

Primarily, the patients were asked about recent intravenous abuse of substances, i.e. specific drugs injected during the preceding 48 hours and the time in hours since the last intravenous injection. In the whole sample, the time intervals since the last intravenous injection were grouped as follows: maximally 24 hours, more than 24 hours but maximally 48 hours, more than 48 hours but maximally one week, more than one week but maximally one month, or more than one month. Patients were divided into two groups according to admitted intravenous substance abuse within the last 48 hours. Group 1 consisted of 17 patients (34 samples) who admitted to intravenous BPN/BNX abuse in maximally 48 hours from sampling. The remaining 33 patients who gave 53 samples formed Group 2.

The patients were also interviewed regarding factors that might interfere with the lactose assay, i.e. whether the patient had diabetes, lactose intolerance, or any intestinal illness, or whether the patient was pregnant or breastfeeding. These factors did not result in exclusion from the study, as this study is currently experimental and the level of lactose concentrations did not lead to any consequences for the patients These interfering factors were also examined in cases where the $\mathrm{N}$ was sufficient for statistical analyses. 
Urine sample collection for mass spectrometric and enzymatic analyses

All patient urine samples were collected under supervision (a one-way mirror). Samples were collected for the analysis by two mass spectrometric methods, consisting of ultra-highperformance liquid chromatography/high-resolution quadrupole time-of-flight mass spectrometry (UHPLC-HR-QTOFMS) ${ }^{17}$ and UHPLC-triple quadrupole mass spectrometry (UHPLC-MS/MS), as part of the clinical work. These methods enabled both screening and quantitative analysis of many illegal and legal psychotropic substances. The quantitative performance for buprenorphine, norbuprenorphine, and naloxone was similar to that in a method described earlier ${ }^{25}$. The same samples were also used for determination of the concentrations of lactose. The laboratory analyses were performed blinded both to the clinical evaluations and to each other.

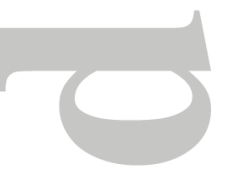

Enzymatic assay for determination of lactose in urine

The urine samples were stored at $-20^{\circ} \mathrm{C}$ until the analysis of lactose. Prior to analysis, the samples were clarified with the use of a 10K centrifugal filter (VWR International, Radnor, PA, USA) and diluted 1:1 with water. One sample was discarded from lactose analysis due to interfering debris in the sample. Sample volume in lactose assay was $30 \mu \mathrm{L}$.

Lactose was analyzed as previously described ${ }^{24}$. The enzymatic R-biopharm Lactose/DGalactose kits were purchased from Roche (Darmstadt, Germany). The assay is based on the enzymatic hydrolysis of disaccharide to monosaccharides. In the lactose assay, further oxidation of galactose and concomitant reduction of $\mathrm{NAD}^{+}$to $\mathrm{NADH}$ is measured by absorbance at $340 \mathrm{~nm}$. The reactions were adapted for a 96-well microplate and the measurement was performed on a Multiscan GO-spectrophotometer (ThermoScientific, 
Waltham, MA, USA). Parallel measurements were performed without the disaccharidase in the reaction to correct the effect of possible monosaccharides in urine. The limit of quantification (LOQ) for the lactose assay is $15 \mathrm{mg} / \mathrm{l}$ as previously described in the validation of the method for urine samples ${ }^{24}$. For statistical analyses, values less than LOQ for lactose was reported as $15 \mathrm{mg} / \mathrm{l}$.

\section{Statistical tools}

The patients were divided into two groups: Group 1 samples $(\mathrm{N}=34)$, which were given by the patients with admitted intravenous abuse of BPN/BNX during the last 48 hours, and Group 2 samples $(\mathrm{N}=53)$, which were given by the patients with a longer time interval since the last injection prior to sample collection. The normality of the distributions of lactose concentration level was analyzed with the Kolmogorov-Smirnov test. Because lactose was not normally distributed, non-parametric tests were applied in further statistical analyses. Data were indicated as medians, lower quartiles, upper quartiles, and range of the values. Two-group comparisons for continuous variables were carried out with the independent samples median test and for nominal variables with Fisher's exact test. The confidence interval (CI) was 95\%, and the statistical significance level was 0.05. Spearman's rho $(\rho)$ correlations were analyzed for lactose concentrations with different variables. The collected data were analyzed by using the IBM SPSS Statistics 25 software. 


\section{Results}

Descriptive data

Descriptive data of patients are presented in Table 1. One urine sample was from a patient with diabetes, 20 samples were from patients with lactose intolerance, and three samples were from patients with an intestinal disorder. None of the patients were pregnant or lactating. All Group 1 urine samples were given by patients who were in evaluation of the need for OMT, in contrast to no urine samples given by OMT patients $(\mathrm{p}<0.01)$. Therefore according to the interview, none of the OMT patients had abused substances intravenously.

Concentrations of lactose and BPN/BNX

The distributions of urine concentrations of lactose are presented in Table 2. The median lactose concentration in Group 1 differed significantly $(\mathrm{p}<0.01)$ from that in Group 2 . In addition, the median buprenorphine concentration in Group 1 differed significantly from that in Group $2(\mathrm{p}=0.034)$. Median norbuprenorphine or naloxone concentrations did not differ significantly when Groups 1 and 2 were compared. The boxplots of lactose concentrations in Groups 1 and 2 are presented in Figure 1.

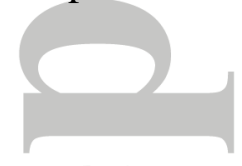

Needle marks

All Group 1 urine samples $(\mathrm{N}=34)$ were given by patients with recent injection marks and seven Group 2 samples were given by patients with recent injection marks ( $p<0.01$ ). Old injection marks were found in 74 samplings from patients; 33 urine samples were given by Group 1 patients and 41 urine samples by Group 2 patients $(\mathrm{p}=0.013)$. 
Correlations between disaccharide concentrations and time from last injection within the 48hour post-injection period

There was a negative correlation between the hours since the last intravenous injection and lactose concentration $(\rho-0,63, \mathrm{p}<0.01)$. The correlation is shown in Figure 2.

Correlations between lactose and OMT medication concentrations

Spearman's rho correlations were analyzed between lactose concentrations with buprenorphine, norbuprenorphine and naloxone concentrations. The correlation of buprenorphine with lactose was 0.594 (significant at the 0.01 level) and its metabolite norbuprenorphine with lactose was 0.362 (significant at the 0.05 level). There was no statistically significant correlation between naloxone and lactose.

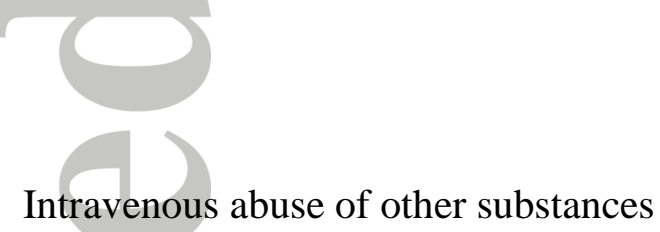

Four patients reported injecting amphetamine within 48 hours, and two of these patients had injected also buprenorphine. Lactose concentration was highest in urine samples given during the first post-injection day and when there was concomitant abuse of BPN/BNX (Table 3). All of these patients had recent injection marks and the presence of the reported abused substances could be verified by the mass spectrometric methods. No traces of amphetamine were found from other samples. The study patients did not report intravenous abuse of any other substances in the 48-hour post-injection period.




Effect of hypolactasia on the analysis method

The median lactose concentration in Group 2 samples obtained from patients with hypolactasia did not differ significantly compared with the samples from patients with no hypolactasia ( $\mathrm{p}=0.451$, independent samples median test). The median urine concentrations of lactose for patients with hypolactasia and the remaining patients in Group 2 were $26 \mathrm{mg} / \mathrm{l}$ and $32 \mathrm{mg} / \mathrm{l}$, respectively. The effect of diabetes and intestinal disorders could not be evaluated statistically due to the small number of patients with these diseases. Only one patient had diabetes accompanied by intestinal disorder (lactose $74 \mathrm{mg} / \mathrm{L}$ ), and one patient had intestinal disorder only, lactose 107 and $71 \mathrm{mg} / \mathrm{L}$ at two different sampling times.

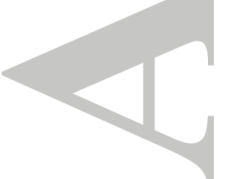

\section{Discussion and conclusions}

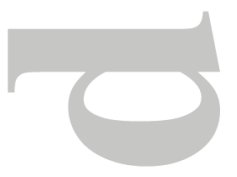

This study shows that the urine lactose is a potential indicator of recent intravenous abuse of BNX among opioid dependent patients. The median urine lactose concentration was significantly higher in Group $1(86 \mathrm{mg} / \mathrm{l})$ than in Group 2 patients $(24 \mathrm{mg} / \mathrm{l})$. All urine samples $(\mathrm{N}=12)$ with lactose concentration more than $140 \mathrm{mg} / \mathrm{l}$ were given by the Group 1 patients. In addition, the lactose concentration correlated significantly with the concentrations of buprenorphine and norbuprenorphine. The lactose concentrations decreased significantly during the 48 -hour post-injection period. Figure 2 shows that the lactose concentrations are highest in the first hours after admitted intravenous injection, decreasing rapidly after 7-10 hours. It appears that most of the lactose is secreted in urine within the first 24 hours.

Intravenous substance abuse is often studied by surveys and also by needle mark examinations. In Finland, the most common intravenously abused illicit drugs are 
amphetamine and buprenorphine ${ }^{26}$. Intravenous substance abuse predisposes the patients to infectious diseases and other somatic complications, causes serious poisonings ${ }^{27-29}$ and impairs the benefits of OMT. To find a diagnostic solution to detect intravenous abuse, Jungen et al. ${ }^{23}$ introduced a liquid chromatography - photodiode array detection (LC-DAD) method to detect urine disaccharides and, more recently, an LC-MS/MS method for the same purpose $^{30}$. While these methods are specific and measure the disaccharides directly, the enzymatic method used in the present study is cost-effective, easy to perform, and readily adapted to every laboratory, as it requires only basic laboratory equipments. The new diagnostic tool may for its part improve the reported underuse of BPN ${ }^{5,7}$. Although the focus here is mainly on BPN/BNX, the assay can be applied to reveal other intravenously abused substances if the excipients include lactose. For example, methadone hydrochloride tablets (Mallinckrodt Inc.) contain lactose monohydrate. Thus, the urine lactose analyses can be useful also in revealing the intravenous abuse of MET in other clinical settings. This is important due to the increasing amount of reported methadone diversion ${ }^{31}$. Furthermore, opioid dependence is a chronic and relapsing disorder ${ }^{3}$ and even after a long abstinence intravenous abuse of substances may occur.

This study reports four samples from patients who had admitted to recent injecting of amphetamine. Two of these patients had injected also BPN/BNX. The highest level of lactose was observed for a patient with intravenous co-abuse of amphetamine and BPN/BPX in the sample provided during the first post-injection day. On the other hand, the lowest concentration of lactose was found in the urine sample of a patient who had abused only amphetamine nearly two days earlier. This finding is in line with the fact that amphetamine preparations may contain lactose in different amounts to dilute or to add bulk to the abused substance $^{18}$. 
In our previous study, we applied an enzymatic method to detect the disaccharides lactose and sucrose mainly in PM urine samples ${ }^{24}$, and a correlation was found between lactose concentration and the presence of BPN or BNX. A similar enzymatic method had been previously used in the assessment of intestinal permeability of patients with hypolactasia, celiac disease, or inflammatory bowel disorders ${ }^{20,21}$ Although the enzymatic method itself is feasible and cost-effective, a major limitation of our previous study was the lack of detailed information concerning the subjects studied, as we had access to laboratory results only, and not to autopsy findings or previous health records. Moreover, clinical samples were tested only to evaluate the effect of PM changes on sample material. In this study, hypolactasia did not appear to elevate lactose concentrations significantly, although it is likely that the patients suffering from hypolactasia avoid lactose-containing food and drinks due to the unpleasant symptoms. Therefore, it is unlikely that disturbing amounts of lactose would be present in urine due to impaired intestinal hydrolysis, if only small amounts or none is ingested. Information on intestinal disorders and hypolactasia was based on patient interview and not confirmed by laboratory methods, which is one limitation of this study. The effect of neither pregnancy nor lactation on urine lactose concentrations could be assessed in this study, as there were no patients in these groups. These factors require additional research with patient samples from gastroenterological and obstetric fields.

One purpose of OMT is reducing intravenous abuse of substances. This decreases the risk of infectious diseases, poisonings, and severe somatic complications. Medications intended for oral use but crushed and diluted in water and then injected as well as injected illicit drugs, like heroin, cocaine, and methadone, commonly contain microscopic insoluble material, such as talc, cellulose, crospovidone, and starch, which can induce even fatal pulmonary vascular injury ${ }^{32,33}$. Prevention of such complications by all possible means is crucial. 
In addition to the limitations discussed before, the numbers of urine samples and study patients were relatively small, giving rise to possible statistical type I and II errors. Moreover, this study cannot definitely state which BPN/BNX preparations were abused by the patients. Regarding new BNX medications, the rapidly dissolving sublingual tablet containing sucralose is not yet officially available in Finland. Furthermore, it is possible that in Group 2 there were some patients that had intravenously abused substances, but were not willing to admit it.

In conclusion, enzymatic detection of lactose in urine can reveal intravenous abuse of some BPN/BNX preparations. In addition to the laboratory analysis, information acquired from patients and examination of needle marks are always needed in clinical practice with patients suspected of intravenous drug abuse.

\section{Acknowledgements}

We thank the Yrjö Jahnsson Foundation for providing research grant to TK. 


\section{References}

1. WHO. Guidelines for the Psychosocially Assisted Pharmacological Treatment of Opioid Dependence, Geneva; 2009.

2. Kenworthy, J, Yi, Y, Wright, A, Brown, J, Maria Madrigal, A, and Dunlop, WCN. Use of opioid substitution therapies in the treatment of opioid use disorder: results of a UK cost-effectiveness modelling study, J Med Econ 2017;20(7):740-748. doi:10.1080/13696998.2017.1325744.

3. Schuckit, MA. Treatment of Opioid-Use Disorders, $N$ Engl J Med 2016;375(16):1596-1597. doi:10.1056/NEJMc1610830.

4. Soyka, M. Treatment of opioid dependence with buprenorphine: current update, Dialogues Clin Neurosci 2017;19(3):299-308. http://www.ncbi.nlm.nih.gov/pubmed/29302227

5. Nielsen, S, Larance, B, and Lintzeris, N. Opioid Agonist Treatment for Patients With Dependence on Prescription Opioids, JAMA 2017;317(9):967-968. doi:10.1001/jama.2017.0001.

6. Skolnick, P. The Opioid Epidemic: Crisis and Solutions, Annu Rev Pharmacol Toxicol 2017; doi:10.1146/annurev-pharmtox-010617-052534.

7. Tsui, JI, Burt, R, Thiede, H, and Glick, SN. Utilization of buprenorphine and methadone among opioid users who inject drugs, Subst Abus 2017;1-6. doi:10.1080/08897077.2017.1363844.

8. Huhn, AS, and Dunn, KE. Why aren't physicians prescribing more buprenorphine?, $J$ Subst Abuse Treat 2017;78(1-7. doi:10.1016/j.jsat.2017.04.005.

9. Alho, H, Sinclair, D, Vuori, E, and Holopainen, A. Abuse liability of buprenorphinenaloxone tablets in untreated IV drug users, Drug Alcohol Depend 2007;88(1):75-78. doi:10.1016/j.drugalcdep.2006.09.012.

10. Bazazi, AR, Yokell, M, Fu, JJ, Rich, JD, and Zaller, ND. Illicit use of buprenorphine/naloxone among injecting and noninjecting opioid users, $J$ Addict Med 2011;5(3):175-180. doi:10.1097/ADM.0b013e3182034e31.

11. Yokell, MA, Zaller, ND, Green, TC, and Rich, JD. Buprenorphine and buprenorphine/naloxone diversion, misuse, and illicit use: an international review, Curr Drug Abuse Rev 2011;4(1):28-41.

http://www.ncbi.nlm.nih.gov/pubmed/21466501

12. Butler, SF, Black, RA, Severtson, SG, Dart, RC, and Green, JL. Understanding abuse of buprenorphine/naloxone film versus tablet products using data from ASI-MV(R) substance use disorder treatment centers and RADARS(R) System Poison Centers, $J$ Subst Abuse Treat 2018;84(42-49. doi:10.1016/j.jsat.2017.10.010.

13. Launonen, E, Alho, H, Kotovirta, E, Wallace, I, and Simojoki, K. Diversion of opioid maintenance treatment medications and predictors for diversion among Finnish maintenance treatment patients, Int J Drug Policy 2015;26(9):875-882. doi:10.1016/j.drugpo.2015.03.007.

14. Heikman, PK, Muhonen, LH, and Ojanpera, IA. Polydrug abuse among opioid maintenance treatment patients is related to inadequate dose of maintenance treatment medicine, BMC Psychiatry 2017;17(1):245. doi:10.1186/s12888-017-1415-y.

15. Launonen, E, Wallace, I, Kotovirta, E, Alho, H, and Simojoki, K. Factors associated with non-adherence and misuse of opioid maintenance treatment medications and intoxicating drugs among Finnish maintenance treatment patients, Drug Alcohol Depend 2016;162(227-235. doi:10.1016/j.drugalcdep.2016.03.017.

16. Heikman, P, Sundstrom, M, Pelander, A, and Ojanpera, I. New psychoactive substances as part of polydrug abuse within opioid maintenance treatment revealed by 
comprehensive high-resolution mass spectrometric urine drug screening, Hum Psychopharmacol 2016;31(1):44-52. doi:10.1002/hup.2512.

17. Sundstrom, M, Pelander, A, Angerer, V, Hutter, M, Kneisel, S, and Ojanpera, I. A high-sensitivity ultra-high performance liquid chromatography/high-resolution timeof-flight mass spectrometry (UHPLC-HR-TOFMS) method for screening synthetic cannabinoids and other drugs of abuse in urine, Anal Bioanal Chem 2013;405(26):8463-8474. doi:10.1007/s00216-013-7272-8.

18. Cole, CJ, L.; McVeigh, J.; Kicman, A.; Syed, Q; Bellis, M. A. (2010) A guide to adulterants, bulking agents and other concomitants found in illicit drugs.

19. Deng, Y, Misselwitz, B, Dai, N, and Fox, M. Lactose Intolerance in Adults: Biological Mechanism and Dietary Management, Nutrients 2015;7(9):8020-8035. doi:10.3390/nu7095380.

20. Hessels, J, Eidhof, HH, Steggink, J, Roeloffzen, WW, Wu, K, Tan, G, van de Stadt, J, and van Bergeijk, L. Assessment of hypolactasia and site-specific intestinal permeability by differential sugar absorption of raffinose, lactose, sucrose and mannitol, Clin Chem Lab Med 2003;41(8):1056-1063. doi:10.1515/CCLM.2003.163.

21. Hessels, J, Snoeyink, EJ, Platenkamp, AJ, Voortman, G, Steggink, J, and Eidhof, HH. Assessment of intestinal permeability: enzymatic determination of urinary mannitol, raffinose, sucrose and lactose on Hitachi analyzer, Clin Chem Lab Med 2003;41(1):33-38. doi:10.1515/CCLM.2003.007.

22. Ekman, B, Wahlberg, J, and Landberg, E. Urine oligosaccharide pattern in patients with hyperprolactinaemia, Glycoconj J 2015;32(8):635-641. doi:10.1007/s10719-0159610-x.

23. Jungen, H, Andresen-Streichert, H, Muller, A, and Iwersen-Bergmann, S. Disaccharides in urine samples as markers of intravenous abuse of methadone and buprenorphine, J Anal Toxicol 2013;37(9):652-658. doi:10.1093/jat/bkt086.

24. Keltanen, T, Mariottini, C, Walta, AM, Rahikainen, AL, and Ojanpera, I. Enzymatic assays for detecting lactose and sucrose in urine to reveal intravenous drug abuse with emphasis on buprenorphine, Drug Test Anal 2017;9(6):949-952. doi:10.1002/dta.2050.

25. Heikman, P, Hakkinen, M, Gergov, M, and Ojanpera, I. Urine naloxone concentration at different phases of buprenorphine maintenance treatment, Drug Test Anal 2014;6(3):220-225. doi:10.1002/dta.1464.

26. EMCDDA. Finland, Country Drug Report 2017.

27. Escuissato, DL, Ferreira, RG, Barros, JA, and Marchiori, E. Pulmonary talcosis caused by intravenous methadone injection, J Bras Pneumol 2017;43(2):154-155. doi:10.1590/S1806-37562016000000337.

28. Hakkinen, M, Heikman, P, and Ojanpera, I. Parenteral buprenorphine-naloxone abuse is a major cause of fatal buprenorphine-related poisoning, Forensic Sci Int 2013;232(1-3):11-15. doi:10.1016/j.forsciint.2013.06.017.

29. Iwersen-Bergmann, S, Jungen, H, Andresen-Streichert, H, Muller, A, Elakkary, S, Puschel, K, and Heinemann, A. Intravenous methadone application as a serious risk factor for an overdose death: methadone-related fatalities in Hamburg from 2007 to 2012, Int J Legal Med 2014;128(5):751-764. doi:10.1007/s00414-014-1017-x.

30. Jungen, H, Andresen-Streichert, H, Muller, A, and Iwersen-Bergmann, S. Monitoring Intravenous Abuse of Methadone or Buprenorphine in Opiate Maintenance Treatment (OMT): A Simple and Fast LC-MS-MS Method for the Detection of Disaccharides in Urine Samples, J Anal Toxicol 2017;41(1):22-31. doi:10.1093/jat/bkw099. 
31. Reddon, H, Ho, J, DeBeck, K, Milloy, MJ, Liu, Y, Dong, H, Ahamad, K, Wood, E, Kerr, T, and Hayashi, K. Increasing diversion of methadone in Vancouver, Canada, 2005-2015, J Subst Abuse Treat 2018;85(10-16. doi:10.1016/j.jsat.2017.11.010.

32. Ganesan, S, Felo, J, Saldana, M, Kalasinsky, VF, Lewin-Smith, MR, and Tomashefski, JF, Jr. Embolized crospovidone (poly[N-vinyl-2-pyrrolidone]) in the lungs of intravenous drug users, Mod Pathol 2003;16(4):286-292. doi:10.1097/01.MP.0000062653.65441.DA.

33. Nguyen, VT, Chan, ES, Chou, SH, Godwin, JD, Fligner, CL, Schmidt, RA, and Pipavath, SN. Pulmonary effects of i.v. injection of crushed oral tablets: "excipient lung disease", AJR Am J Roentgenol 2014;203(5):W506-515. doi:10.2214/AJR.14.12582.
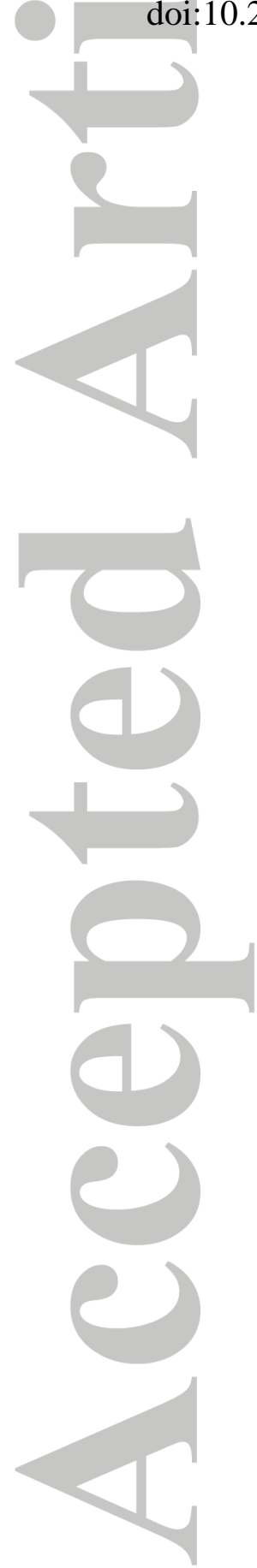


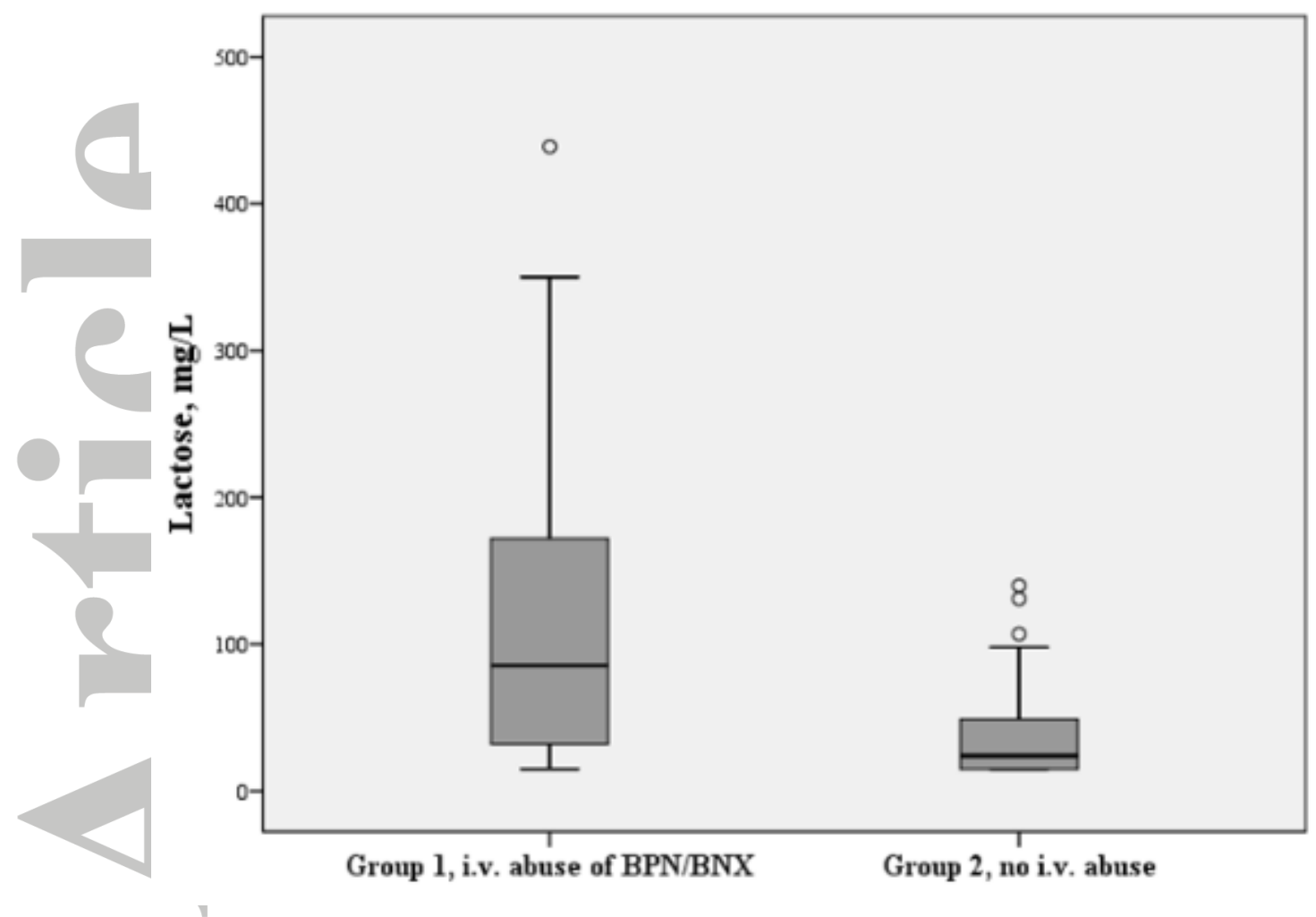

Figure 1. Boxplots of lactose concentrations in Groups 1 (intravenous abuse of BPN/BNX during the last 48 hours) and 2 (no intravenous abuse within 48 hours). 


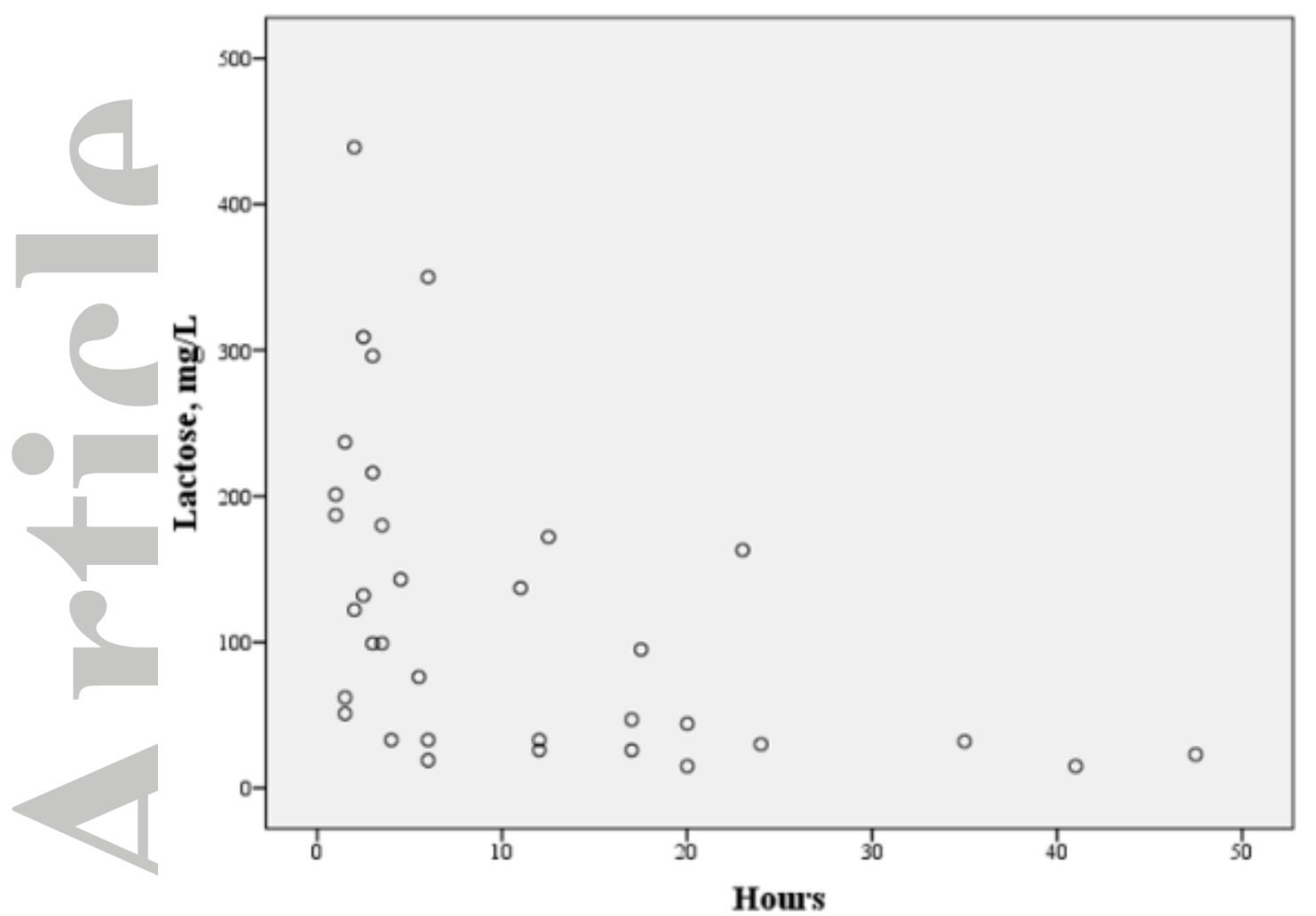

Figure 2. Lactose concentrations in cases with intravenous BPN/BNX abuse relative to time

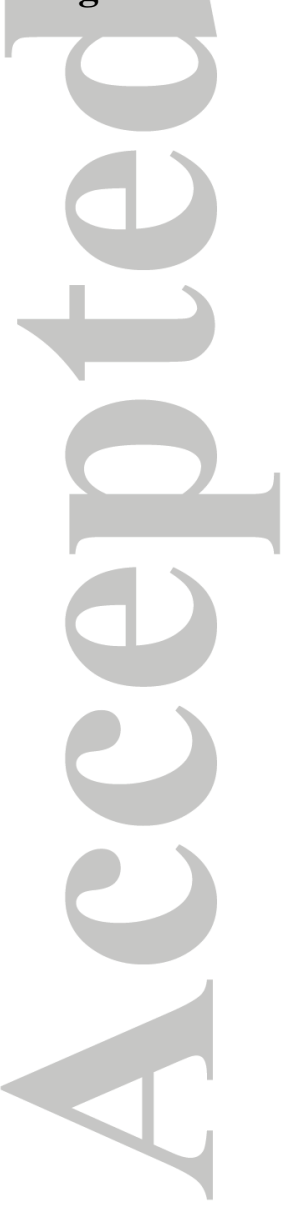
from injection within the 48 -hour post-injection period. 
Table 1. Descriptive patient and sample data.

\begin{tabular}{l|c|c|c}
\hline All & Group 1 & Group 2 \\
\hline Patients & 50 & 17 & 33 \\
Gender (male/female) & $31 / 19$ & $14 / 3$ & $17 / 16$ \\
Age, median (years) & 33 & 31 & 34 \\
Age, range (years) & $21-49$ & $23-37$ & $21-49$ \\
Hypolactasia & 13 & 3 & 10 \\
Diabetes & 1 & 0 & 1 \\
Intestinal disorder & 2 & 0 & 2 \\
Admitted recent i.v. abuse $(\leq 48$ h) & 19 & 17 & 2 \\
\hline BPN or BNX /+amphetamine & $15 / 2$ & $15 / 2$ & 0 \\
\hline Intestinal disorder & 2 & 0 & 2 \\
\hline Admitted recent $(\leq 48$ h) i.v. abuse & $32 / 2$ & $32 / 2$ & 0 \\
\hline
\end{tabular}

BNX; buprenorphine-naloxone

BPN; buprenorphine 
Table 2. Urine concentrations of lactose between intravenous BPN/BNX abusers and other patients

\section{Lactose (mg/l)}

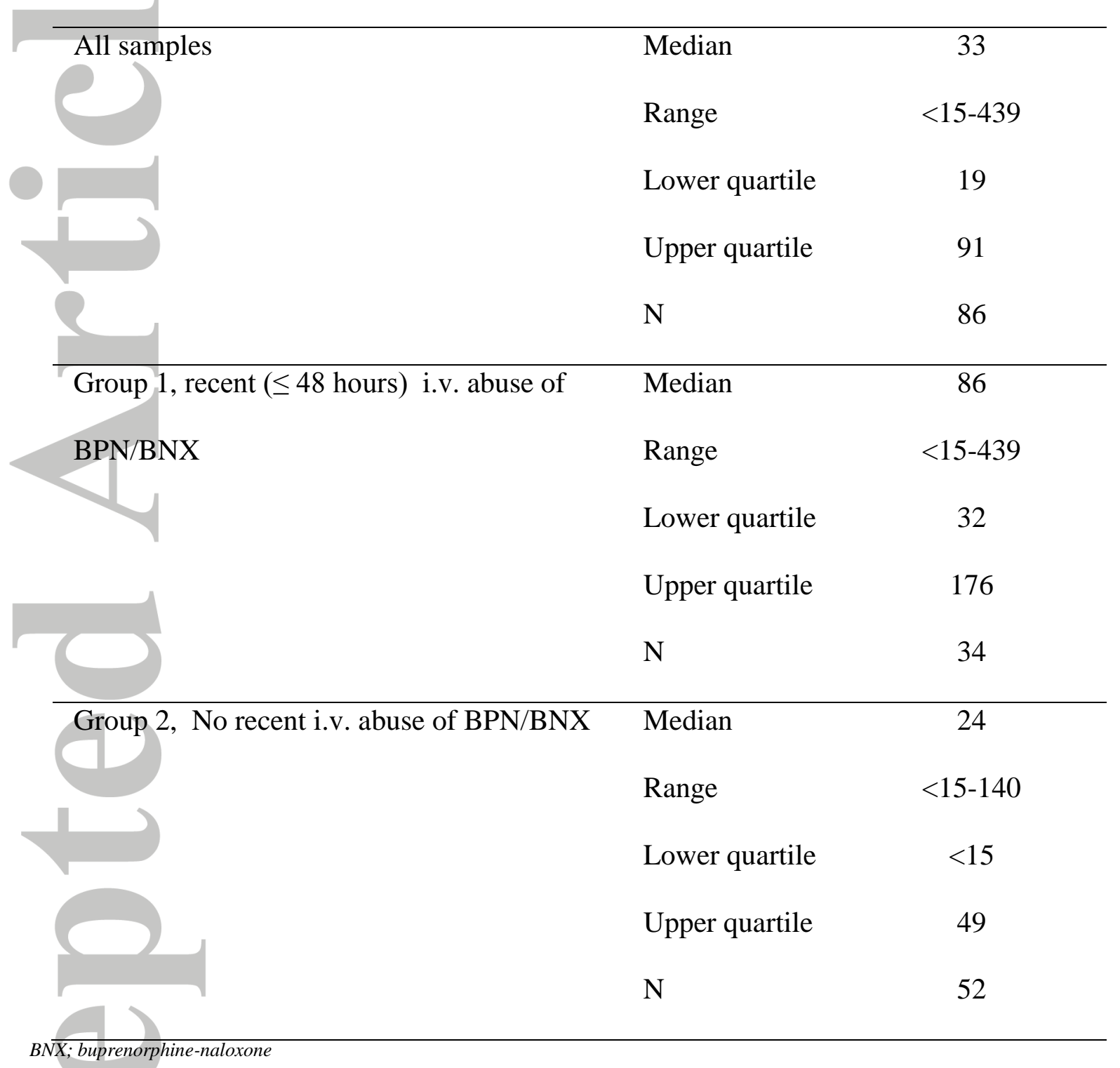

BPN; buprenorphine 
Table 3. Lactose concentrations in samples with intravenous amphetamine abuse.

\begin{tabular}{llll}
\hline $\begin{array}{l}\text { Patient } \\
\text { Admitted substance abuse }\end{array}$ & $\begin{array}{l}\text { Lactose } \\
\text { Amphetamine/Buprenorphine }\end{array}$ & mg/l & intravenous injection \\
\hline $\mathbf{1}$ & yes/yes & 163 & 23 \\
\hline $\mathbf{2}$ & yes/yes & 44 & 20 \\
\hline $\mathbf{3}$ & yes/no & 25 & 31 \\
\hline $\mathbf{4}$ & yes/no & $<15$ & 46 \\
\hline
\end{tabular}




\section{Graphical Abstract}

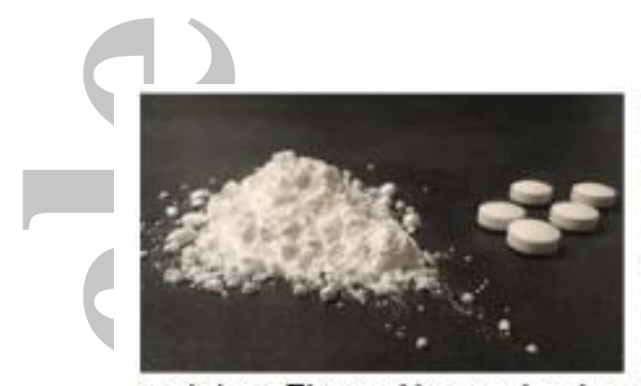

Enzymatic Assay for Urine Lactose in Assessment of Recent Intravenous Abuse of Buprenorphine

Terhi N. Keltanen", Perti K. Heikm an, Leea H. Muhonen, Teemu O. Gunnar, Ilkka A. Ojanperä

Urine samples were analyzed for lactose to investigate if elevated lactose concentrations indicate recent $(<48 \mathrm{~h})$ intravenous abuse of substances containing lactose as excipient. Elevated lactose levels were found in samples given by patients who had recently injected substances intravenously, verified by fresh injection marks. Urine lactose assay can support clinical and toxicological findings when assessing substance abuse.
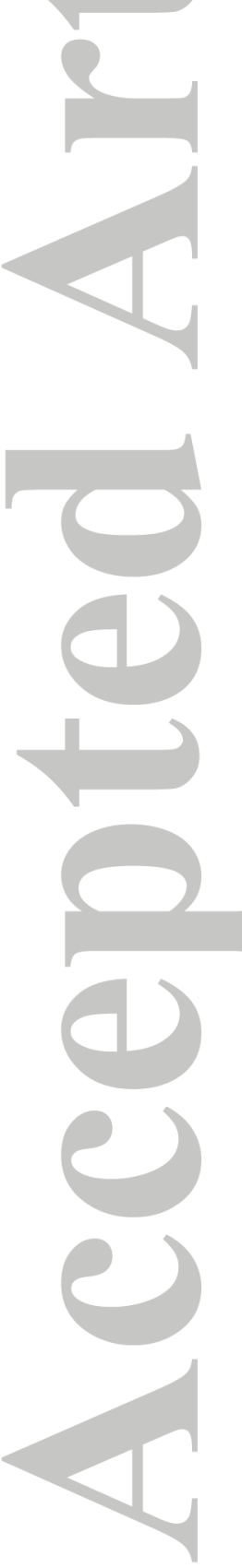